\section{Splicing for survival}

The oncogene $B C R-A B L 1$ hijacks a $B$ cell signaling protein by inducing the synthesis of a linker protein, according to Feldhahn and colleagues on page 1837. This study helps explain how BCR-ABL1 causes B cell leukemias in humans and identifies a potential new target for drug development.

Translocation events that combine unrelated chromosomal sequences are found in up to $65 \%$ of human acute leukemias. One such translocation event creates a chimeric protein between the signaling protein $B C R$ and the tyrosine kinase $A B L 1$. The $B C R-A B L 1$ fusion allows precursor $B$ cells to bypass survival signals from the pre-B cell receptor that are normally required for $B$ cell survival. But exactly how BCR-ABL1 ensures $B$ cell survival in the absence of pre-B cell receptor signals was not completely clear.

Feldhahn et al. now show that Bruton's tyrosine kinase (BTK)-a key component of the pre-B cell receptor signaling pathway-is perpetually

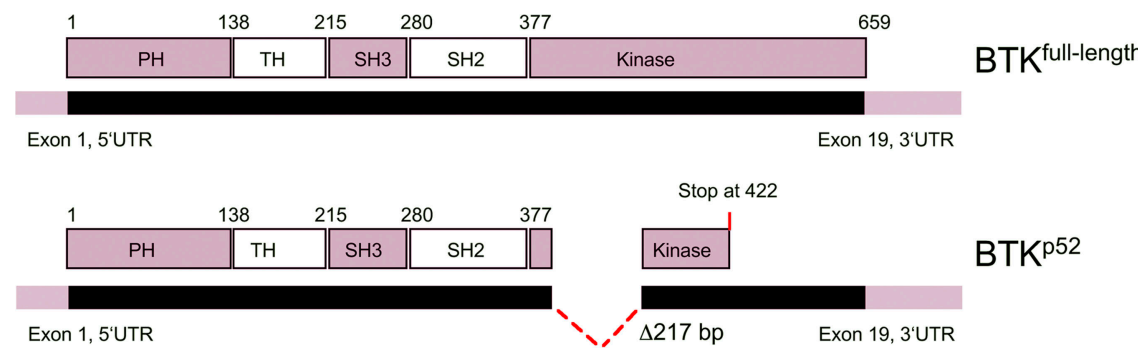

The oncogene BCR-ABL1 induces the synthesis of a splice variant of Bruton's tyrosine kinase $\left(B K^{\mathrm{p} 52}\right)$ that hijacks full-length BTK and transforms B cells.

phosphorylated (and thus activated) in BCR-ABL1-expressing leukemia cells. Downstream signals, including calcium flux and expression of the pro-survival protein $\mathrm{Bcl}-\mathrm{xL}$, were also activated in these cells. BTK phosphorylation depended on the kinase activity of BCR-ABL1, as inhibiting this kinase decreased BTK phosphorylation. But the mechanism was mysterious, as previous studies had ruled out a direct interaction between BCR-ABL1 and BTK.

The authors had noted that several kinase-deficient splice variants of BTK accumulated in leukemia cells. One of these variants $\left(\mathrm{BTK}^{\mathrm{p} 52}\right)$ bound to both BCL-ABL1 and full-length BTK, providing a bridge that allowed $\mathrm{BCR}-$ $A B L 1$ to phosphorylate full-length BTK. $\mathrm{BTK}^{\mathrm{p} 52}$ was needed to maintain cell survival, as removal of this protein drove the cells into apoptosis. Remarkably, the $B C R-A B L 1$ protein itself triggered the production of the BTK splice variants, a finding consistent with previous studies showing that $B C R-A B L 1$ induces the expression of proteins involved in RNA splicing. JEM

\title{
Imaging tolerance
}

The behavior of naive $\mathrm{T}$ cells in the lymph nodes does not betray their fate, according to new footage from live lymph nodes. On page 1815, Zinselmeyer and colleagues show that the movements of $\mathrm{CD}^{+}{ }^{+} \mathrm{T}$ cells destined for tolerance differ only subtly from those destined for priming, suggesting that tolerance cannot be explained by an overt failure of the $\mathrm{T}$ cells to stop so that they can receive a priming signal.

Intravital imaging technology has revealed that naive $\mathrm{T}$ cells dart rapidly around lymph nodes in search of their antigen, briefly contacting antigen presenting cells (APCs) as they go. An encounter with a cognate antigen delivers a stop signal that triggers stable T cell-APC interactions. When this encounter breaks up, the $\mathrm{T}$ cells resume their frenetic activity.
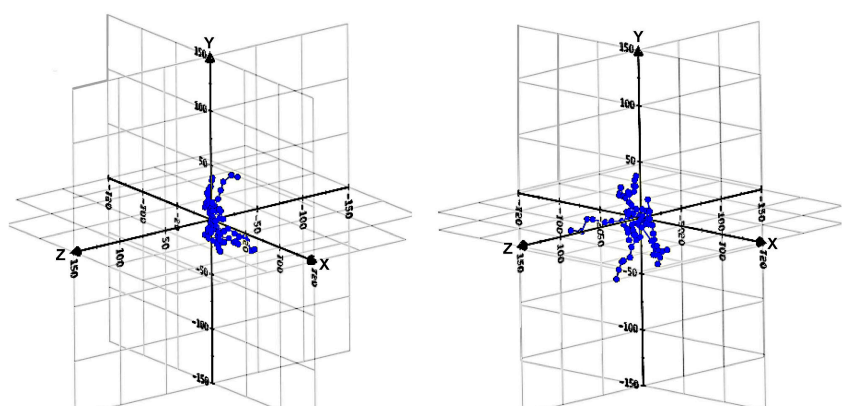

3-dimensional plots of $\mathrm{CD}^{+}{ }^{+} \mathrm{T}$ cell movement in lymph nodes during priming (left) or tolerizing (right) conditions uncover only subtle differences.
Interactions between T cells and APCs may, under certain conditions, lead to T cell tolerance instead of priming. Disruption of the choreographed $\mathrm{T}$ cell behavior in the lymph node is one possible explanation for the distinct functional outcomes. This idea was supported by a recent study showing that $\mathrm{CD}^{+} \mathrm{T}$ cells failed to form stable interactions with dendritic cells under tolerizing conditions, suggesting that the lack of a prolonged signal from the APC may lead to T cell tolerance.

Zinselmeyer et al. now show, however, that $\mathrm{CD}^{+} \mathrm{T}$ cells in lymph nodes of mice fed antigen with or without adjuvant (to induce priming or tolerance) behaved similarly. Under both conditions, naive $\mathrm{T}$ cells formed stable clusters with APCs, although the clusters were smaller and shorterlived under tolerizing conditions. The authors suggest that subtle differences in $\mathrm{T}$ cell clustering, and perhaps the quality of the signal delivered by the APC-rather than the complete presence or absence of a stable interaction-may decide the fate of the $\mathrm{T}$ cell.

Shakhar and colleagues, reporting in Nature Immunology (doi:10.1038/ni1210), also found only subtle differences in $\mathrm{CD}^{+}{ }^{+} \mathrm{T}$ cell behavior in priming or tolerizing conditions. They found that stable T-DC interactions were predictive of $T$ cell activation and proliferation, but not tolerance induction. They speculate that late, dynamic T cell-APC interactions that occur after the $\mathrm{T}$ cells resume their motion may deliver the fate-deciding signal. JEM 


\section{The mark of T reg cells}

Regulatory $\mathrm{T}$ ( $\mathrm{T}$ reg) cells found in the synovial fluid of inflamed joints can be identified by the coexpression of CD4, CD25, and CD27, according to Ruprecht and colleagues on page 1793.

Suppression of damaging $\mathrm{T}$ cell responses by $\mathrm{CD}^{+} \mathrm{T}$ reg cells is critical for the prevention of autoimmune disease. But studying these cells in the context of disease has been problematic, largely because $\mathrm{CD}^{+}{ }^{+} \mathrm{T}$ reg cells and activated $\mathrm{CD}^{+} \mathrm{T}$ cells express many of the same surface molecules and are thus difficult to distinguish. Although coexpression of CD4 and the high affinity interleukin (IL)-2 receptor (CD25) identifies $\mathrm{T}$ reg cells in the circulation, CD25 cannot distinguish between $\mathrm{T}$ reg cells and local effector $\mathrm{T}$ cells, which up-regulate this molecule upon activation.

Ruprecht et al. now show that $\mathrm{CD}^{+}$ CD2 $5^{+}$T reg cells found in the inflamed joints of children with autoimmune arthritis can be distinguished from their $\mathrm{CD} 4{ }^{+} \mathrm{CD} 25^{+}$effector $\mathrm{T}$ cell counterparts by the expression of the TNF receptor family member CD27-a molecule that is down-regulated on activated $\mathrm{T}$ cells. CD27 expression identified T reg cells in these patients, as the ability to suppress $\mathrm{T}$ cell proliferation in vitro resided solely in the $\mathrm{CD} 4{ }^{+} \mathrm{CD} 25^{+} \mathrm{CD} 27^{+} \mathrm{T}$ cell subset.

But why does an autoimmune response develop in these patients despite an abundance of $\mathrm{T}$ reg cells at the site of disease? The authors suggest that the cytokines IL-7 and IL-15, which were detected in the patients' synovial fluid, might be to blame, as the combination of these cytokines reversed the in vitro ability of the $\mathrm{T}$ reg cells to inhibit $\mathrm{T}$ cell proliferation. JEM

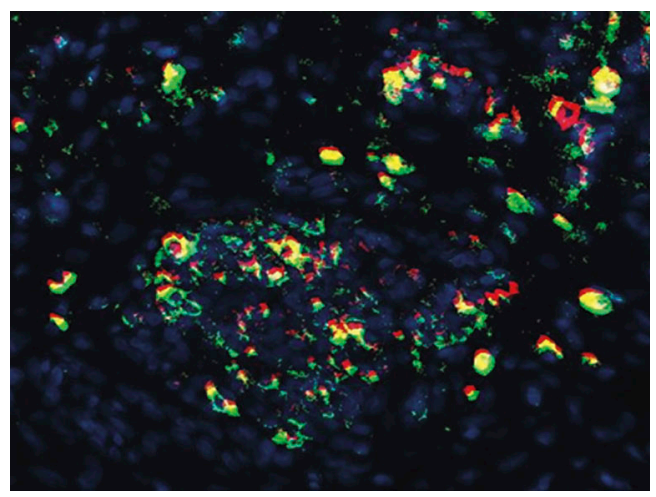

$\mathrm{CD}^{+}{ }^{+}$regulatory $\mathrm{T}$ cells in the synovial tissues of a juvenile arthritis patient coexpress CD25 (red) and CD27 (green).

\section{Brain-tethered T cells}

On page 1805, Kawakami et al. show that antigen-specific $\mathrm{CD} 4^{+} \mathrm{T}$ cells that attack the brain become stationary within brain tissue, whereas nonspecific T cells cruise through without stopping. These intravital images-the first to capture T cells launching an autoimmune attack on the brain-suggest that antigenspecific T cells behave similarly in dense brain tissue as they do in lymph nodes.

Activated $\mathrm{CD} 4^{+} \mathrm{T}$ cells specific for the endogenous brain protein myelin basic protein (MBP) trigger fatal encephalomyelitis in a rat model of multiple sclerosis. These cells migrate into the central nervous system (CNS) where they become reactivated.

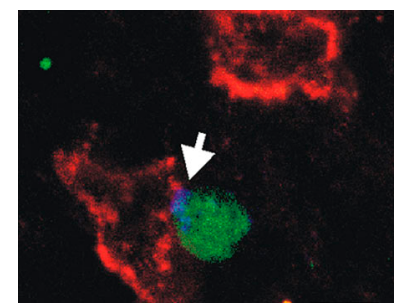

A subset of MBP-specific T cells (green) become stationary in the brain during EAE, presumably after engaging their antigen on antigen-presenting cells (red).
Previous studies by this group showed that both antigen-specific and nonspecific T cells gain access to the CNS, but only specific cells become reactivated once there. Kawakami et al. now show that a subset of MBP-specific T cells-likely those that have encountered antigen-stopped moving inside the brain, appearing tethered to a fixed point. T cells specific for a control antigen kept moving, suggesting that the presence of antigen was required for T cells to stop moving and to trigger disease.

The authors were surprised to find that T cells move as rapidly through the dense brain tissue as in the more aqueous environment of the lymph nodes. How they do this is not known but the authors suggest that the T cells may produce proteases that help clear a path through the compact tissue. JEM

\section{Signaling commitment}

A histone modification thought by many to be a crude bulldozer can contribute to the fine molding of cell behavior, according to a study on page 1825. Rossig et al. show that histone deacetylase (HDAC) activity is required for adult progenitor cells to commit to the endothelial cell lineage. In these cells, HDAC activity was specifically required for the induction of the transcription factor HoxA9, which may be a master regulator of endothelial cell commitment.

HoxA9 was able to drive cell differentiation due to its ability to activate the expression of endothelial cell-specific genes, including endothelial cell nitric oxide synthase (eNOS). In addition, HoxA9deficient mice were unable to recover from ischemic injury, a process that requires formation of new endothelial cells from circulating progenitors.

But the involvement of HDACs in this process may seem counterintuitive, as histone deacetylation of gene promoter elements is normally associated with transcriptional silencing. Indeed, recent reports showed that eNOS expression itself is suppressed by HDAC activity in nonendothelial cells.

The protein complex responsible for activating HoxA9 expression was, however, recently shown to contain HDAC proteins. HDACs can modify nonhistone proteins, including transcription factors, and the acetylation or deacetylation state of these factors can influence target gene activation. The authors suspect that modification of nonhistone proteins may govern the induction of HoxA9. JEM 